\title{
Peripherally Modified Tetraphenylethene: Emerging as a Room-Temperature Luminescent Disc-Like Nematic Liquid Crystal
}

\author{
Jiahua Li,${ }^{\dagger}$ Jia-Hui Wang, ${ }^{\dagger}, \dot{\dagger}$ Xiang-Jian Cao, ${ }^{\dagger},+$ Xiao-Dong Li, ${ }^{\dagger}$ Xiang-Kui Ren $^{*}, \stackrel{+}{*}$ and Zhen-Qiang \\ $\mathrm{Yu}^{*}, \dagger$ \\ $\uparrow$ College of Chemistry and Environmental Engineering, Institute of Low-Dimensional Materials Genome Initiative, \\ Shenzhen University, Shenzhen 518071, P. R. China \\ \$ School of Chemical Engineering and Technology, Tianjin University, Tianjin 300072, P. R. China \\ E-mail: zqyu@szu.edu.cn \\ renxiangkui@tju.edu.cn
}




\section{Table of Content}

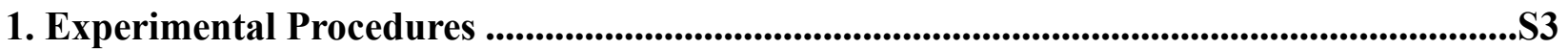

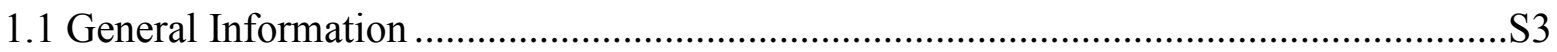

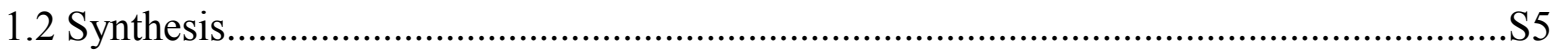

2. Supplementary Figures ....................................................................................................................56

2.1 Optimized Molecular Conformation of Compound 1 ……………................................S6

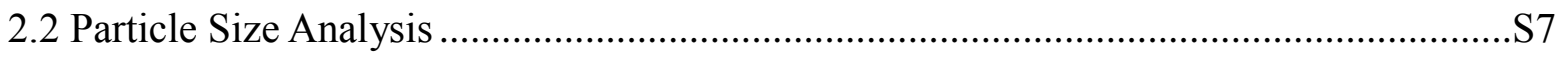

2.3 Spectroscopic Studies of Compounds $\mathbf{1}$ and $\mathbf{2}$ and Their Mixtures ................................S8

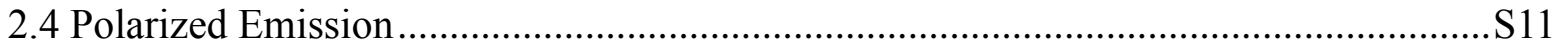

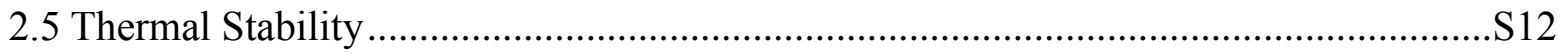

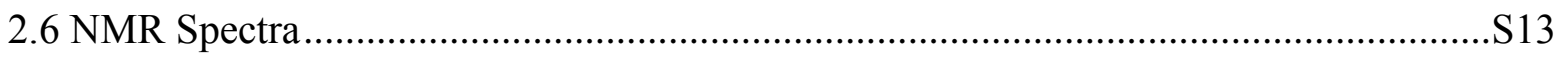

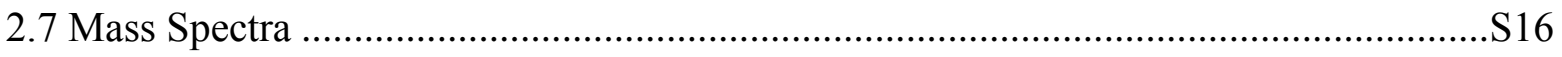

References ..............................................................................................................................................................S17 


\section{Experimental Procedures}

\subsection{General Information}

THF was purchased from XiLong Scientific, and was distilled from sodium metal / benzophenone under $\mathrm{N}_{2}$ flow right prior to use. 1,4-Dioxane, used for spectroscopic experiments, was purchased from Acros Organics in extra pure grade, and was also distilled from sodium metal / benzophenone right prior to use. 1-Bromohexane was purchased from Energy Chemical. Other reagents were purchased from Beijing chemical works and Alfa Aesar.

NMR data were acquired on a Varian VNMRS 400 spectrometer with an Autosw probe or a Bruker AVANCE III 600M with a cryogenic probe. Proton signals were quoted to TMS $(\delta=0.00$ ppm) and ${ }^{13} \mathrm{C}$ signals were referenced to the recommended shift values ${ }^{1}$ of the used solvents $\left(\delta_{\mathrm{CDCl}_{3}}=77.16 \mathrm{ppm}, \delta_{\mathrm{CD}_{2} \mathrm{Cl}_{2}}=53.84 \mathrm{ppm}\right)$. To gain more knowledge about the carbon multiplicity and to improve the signal-to-noise ratio, attached proton test (APT) experiments were conducted, taking the place of the conventional carbon acquisitions. The data were processed with VnmrJ 4.2A or MestReNova. DSC curves were measured on a NETZSCH DSC 200 F3 calorimeter. The sample was first annealed at $110{ }^{\circ} \mathrm{C}$ to fully erase the thermal history prior to subsequent scans. Thermogravimetric data were collected on a TA Q50 thermogravimetric analyser at a heating rate of $10 \mathrm{~K} / \mathrm{min}$ under $\mathrm{N}_{2}$. High-resolution ESI mass spectra were collected on a Bruker Apex IV Fourier-transform spectrometer. MALDI-TOF mass spectra were acquired on a Bruker UltrafleXtreme MALDI-TOF mass spectrometer and $\alpha$-cyano-4-hydroxycinnamic acid was employed as the matrix. UV-Vis absorption spectra were measured on a Varian Cary 4000 spectrometer. The light source was set to change at the wavelength of $350 \mathrm{~nm}$. Room temperature photoluminescence (PL) experiments were conducted on an Agilent Cary Eclipse fluorescence spectrophotometer for solution at RT. Temperature-dependent PL experiments and those required special accessories were performed on a Hitachi F7000 spectrophotometer. Absolute quantum yield was measured on an Edinburgh FLS 980 spectrophotometer at RT. The particle size distribution was assessed on a Beckman DelsaMax CORE light scattering analyzer. Deionized 
water and 1,4-dioxane for the DLS measurements were filtered through millipore filters before use. 1D-WAXD and 2D-WAXD spectra were acquired on a Philips X'Pert Pro diffractometer and a Bruker D8 Discover with GADDS detector, respectively. POM images were captured on a Leica DMLP microscope. 


\subsection{Synthesis}

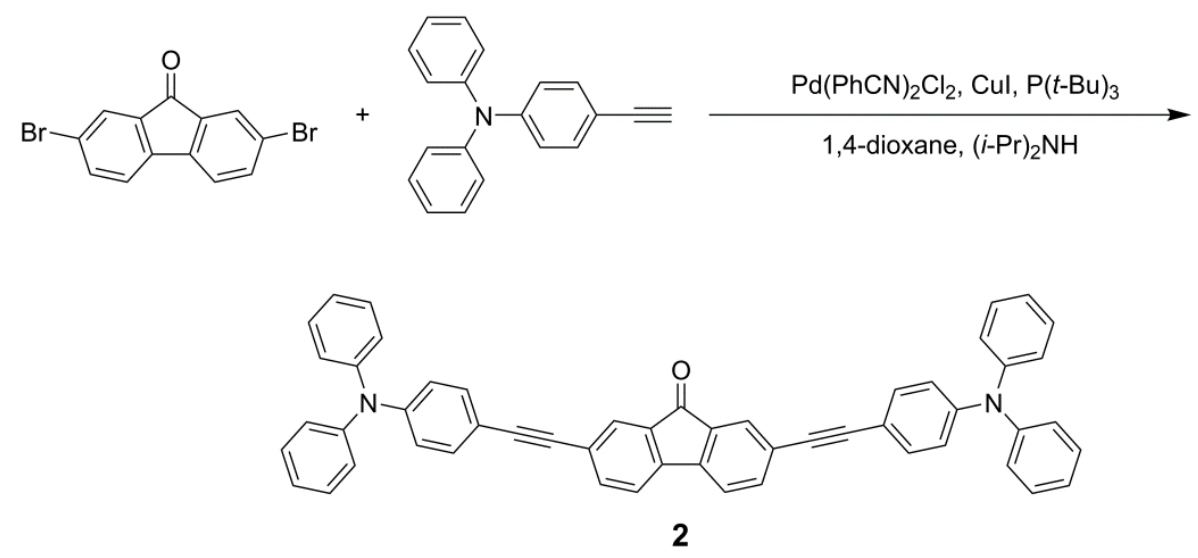

Scheme S1. Synthetic route of the red dye 2.

Compound 2. This compound was prepared via a room-temperature Sonogashira coupling, following Hundertmark's protocol. ${ }^{2}$ Column chromatography (petroleum ether/chloroform $=5 / 1$ ) afforded the pure produce as a red powder in $47 \%$ yield. ${ }^{1} \mathrm{H}$ NMR $\left(600 \mathrm{MHz}, \mathrm{CDCl}_{3}\right) \delta 7.81-$ $7.74(\mathrm{~s}, 2 \mathrm{H}), 7.62(\mathrm{dd}, J=7.7,1.5 \mathrm{~Hz}, 2 \mathrm{H}), 7.49$ (d, $J=7.8 \mathrm{~Hz}, 2 \mathrm{H}), 7.41-7.34$ (m, 4H), $7.32-$ $7.26(\mathrm{~m}, 8 \mathrm{H}), 7.16-7.09(\mathrm{~m}, 8 \mathrm{H}), 7.07(\mathrm{t}, J=7.4 \mathrm{~Hz}, 4 \mathrm{H}), 7.04-6.98(\mathrm{~m}, 4 \mathrm{H}) .{ }^{13} \mathrm{C}$ NMR $(100$ $\left.\mathrm{MHz} \mathrm{CDCl}_{3}\right) \delta 192.53,148.36,147.19,143.00,137.63,134.53,132.76,129.56,127.32,125.24$, 124.90, 123.83, 122.14, 120.58, 115.45, 92.06, 88.10. HRMS (ESI): $\mathrm{C}_{53} \mathrm{H}_{35} \mathrm{~N}_{2} \mathrm{O}: 714.2666$ [M] $]^{+}$; found: 714.2671. 


\section{Supplementary Figures}

\subsection{Optimized Molecular Conformation of Compound 1}

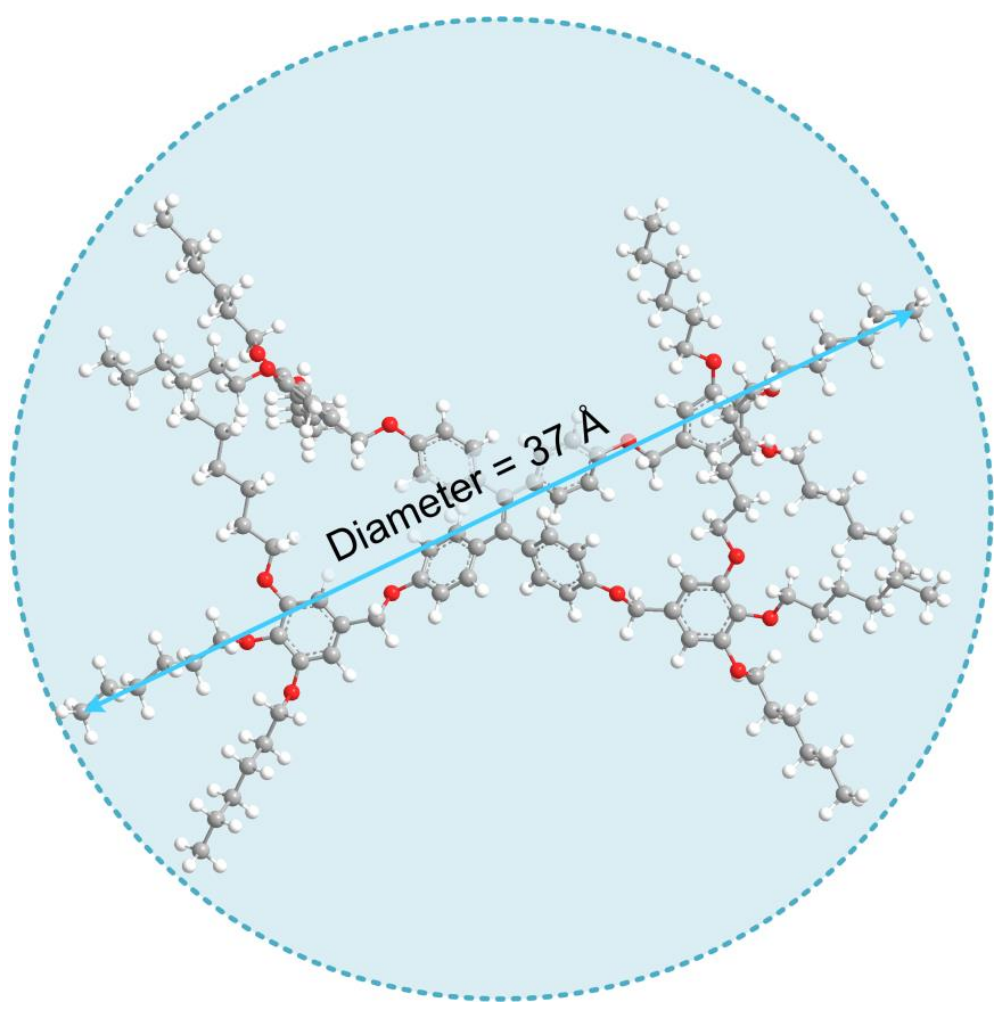

Figure S1. The optimized molecular conformation of compound 1. The optimization was conducted with MM2 method in Chem3D 17.0. All alkyl chains adopted an extended zig-zag conformation. 


\subsection{Particle Size Analysis}
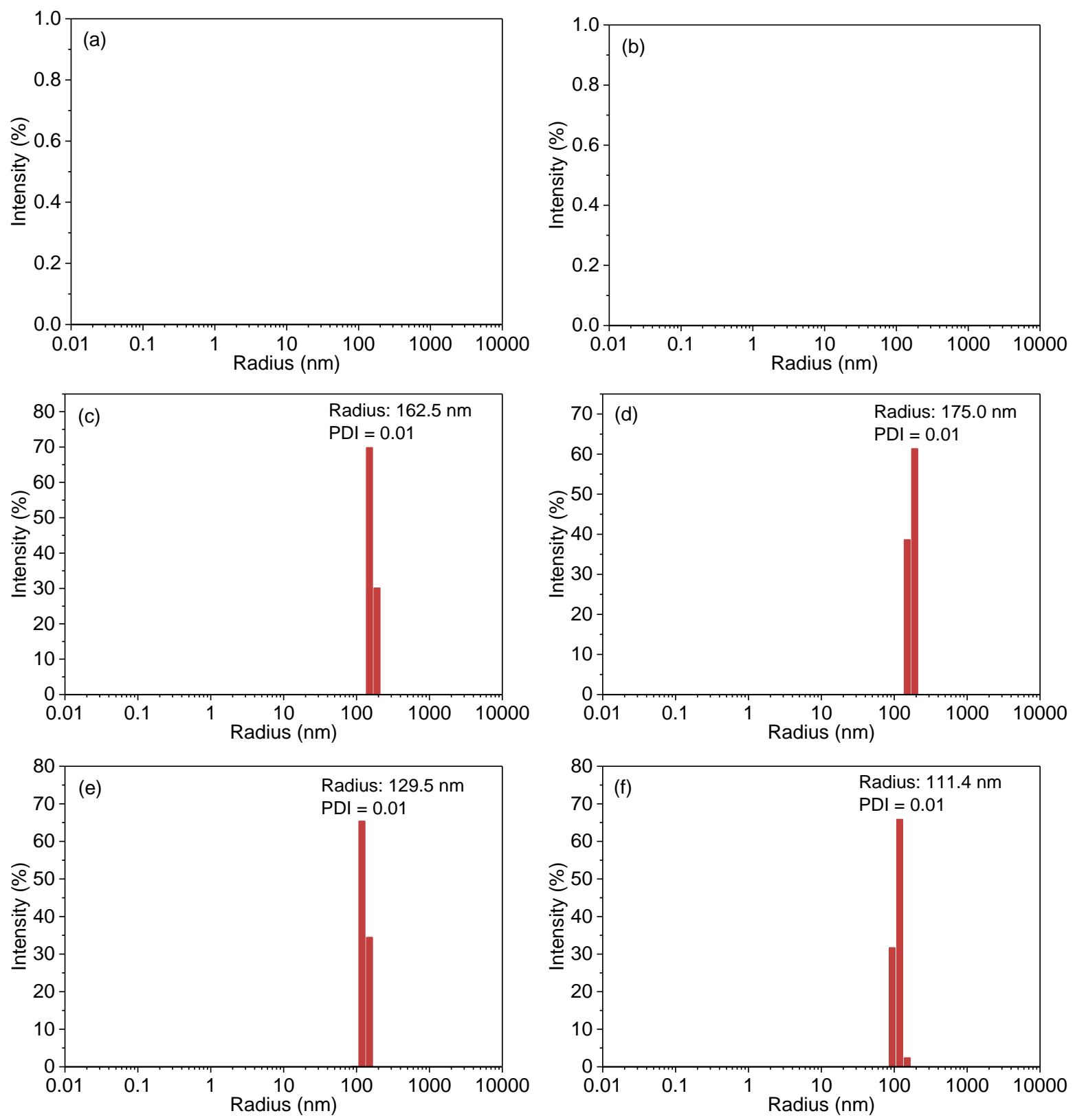

Figure S2. Particle size distributions of compound 1 in the mixture of 1,4-dioxane and water where $f_{\mathrm{w}}=$ (a) $0 \%$, (b) $20 \%$, (c) $40 \%$, (d) $60 \%$, (e) $80 \%$ and (f) $90 \%$. 


\subsection{Spectroscopic Studies of Compounds 1 and 2 and Their Mixtures}

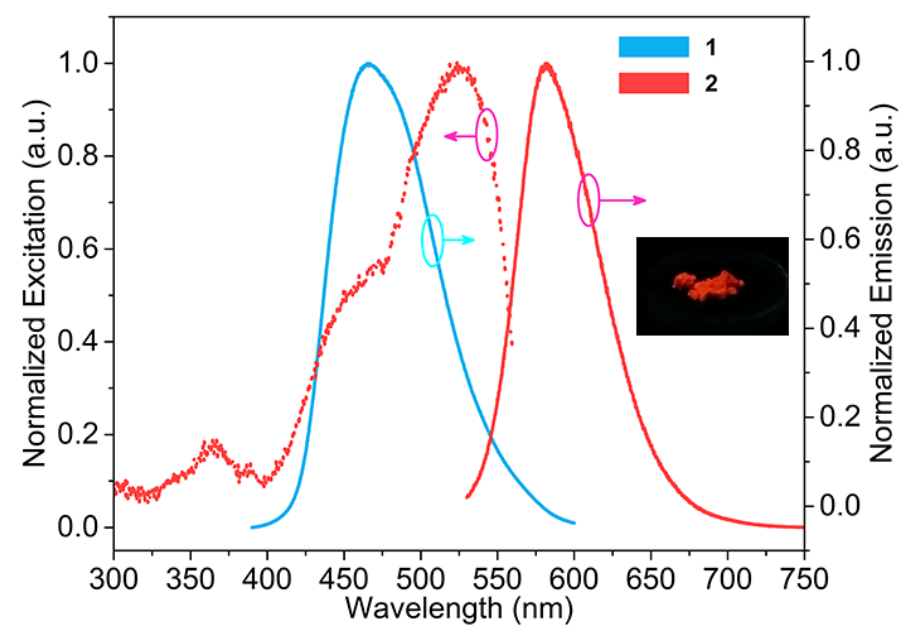

Figure S3. Normalized excitation spectrum $\left(\lambda_{\mathrm{em}}=581 \mathrm{~nm}\right)$ of powder 2 and normalized emission spectra of neat $1\left(\lambda_{\mathrm{ex}}=365 \mathrm{~nm}\right)$ and $\mathbf{2}\left(\lambda_{\mathrm{ex}}=519 \mathrm{~nm}\right)$. 
(a)

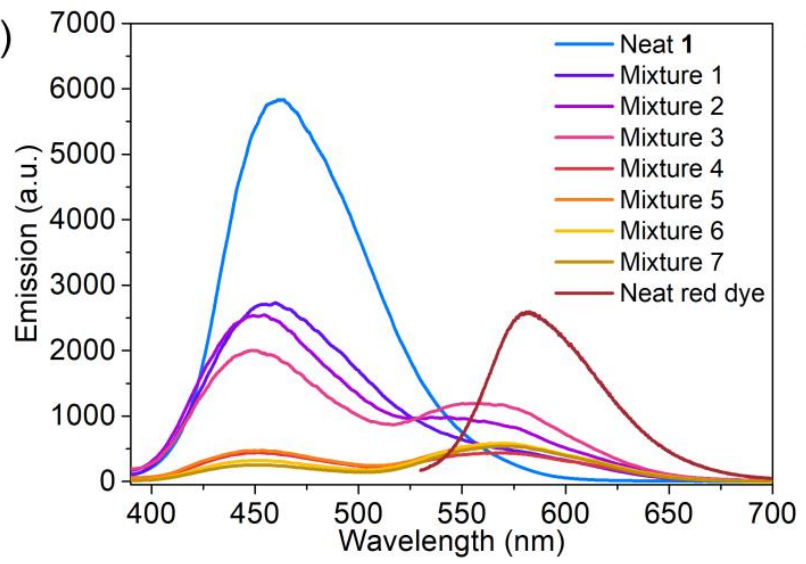

(b)

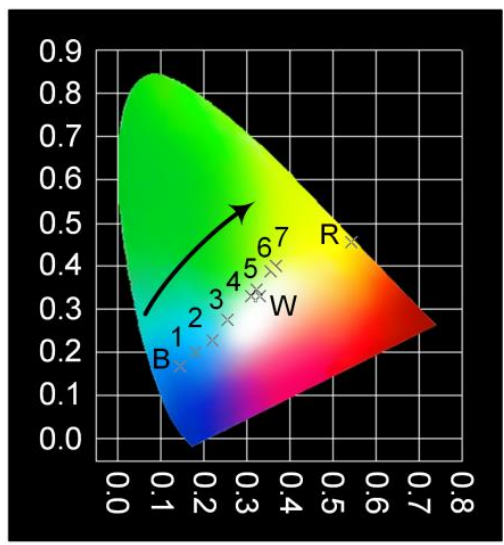

Figure S4. (a) PL spectra and (b) the corresponding CIE diagram of neat 1, all mixtures and pure compound 2. Points $\mathrm{B}, \mathrm{R}$ and $\mathrm{W}$ denote the emission of compounds $\mathbf{1 , 2}$ and the standard WLE with a coordinate of $(0.33,0.33)$, respectively.

Table S1. Summarized information regarding compounds $\mathbf{1}$ and $\mathbf{2}$ and their mixtures

\begin{tabular}{lll}
\hline \multicolumn{1}{c}{ Sample } & \multicolumn{1}{c}{ CIE Coordinate } & \multicolumn{1}{c}{$\boldsymbol{x}_{\mathbf{R}} *$} \\
\hline Neat 1 & $(0.144,0.168)$ & 0.000 \\
Mixture 1 & $(0.180,0.200)$ & 0.0416 \\
Mixture 2 & $(0.219,0.227)$ & 0.0821 \\
Mixture 3 & $(0.256,0.275)$ & 0.0870 \\
Mixture 4 & $(0.309,0.330)$ & 0.163 \\
Mixture 5 & $(0.322,0.346)$ & 0.174 \\
Mixture 6 & $(0.354,0.387)$ & 0.212 \\
Mixture 7 & $(0.369,0.400)$ & 0.262 \\
Neat 2 & $(0.550,0.449)$ & 1.00
\end{tabular}

$* x_{\mathrm{R}}=n_{\mathrm{R}} /\left(n_{\mathrm{R}}+n_{\mathrm{B}}\right)$, where $n_{\mathrm{B}}$ and $n_{\mathrm{R}}$ refer to the amount of compounds $\mathbf{1}$ and $\mathbf{2}$ in mole, respectively. 
(a)

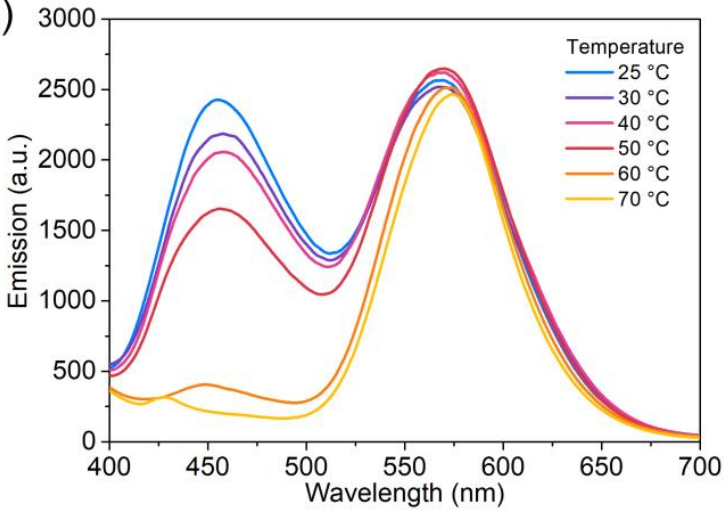

(b)

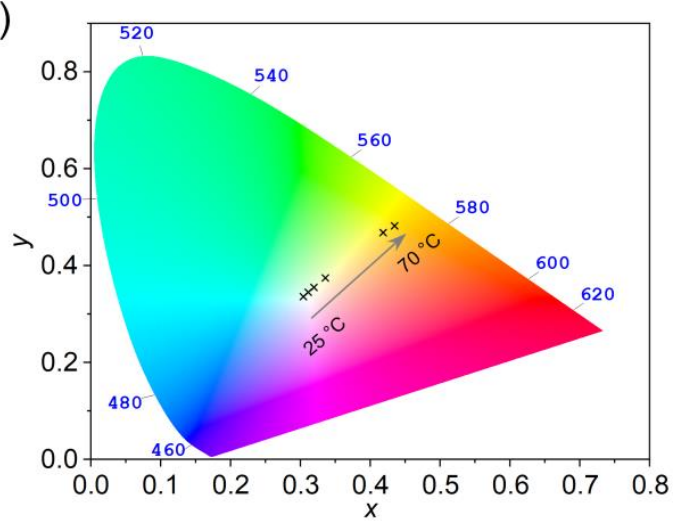

Figure S5. (a) Temperature-dependent PL spectra of the WLE thin film acquired from RT to $70{ }^{\circ} \mathrm{C}$. (b) Corresponding CIE diagram.

Figure S5b showed that the film still retained its WLE properties even if the temperature reached $40{ }^{\circ} \mathrm{C}$. 


\subsection{Polarized Emission}

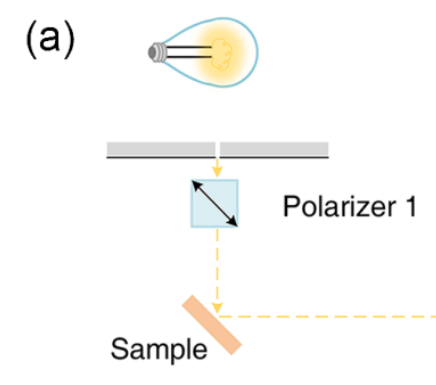

(b)

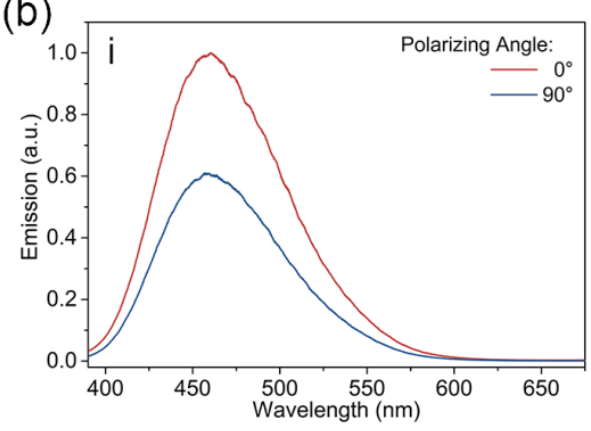

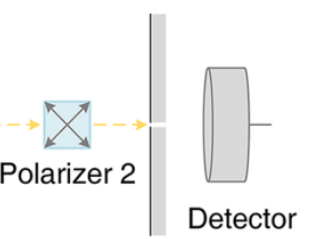

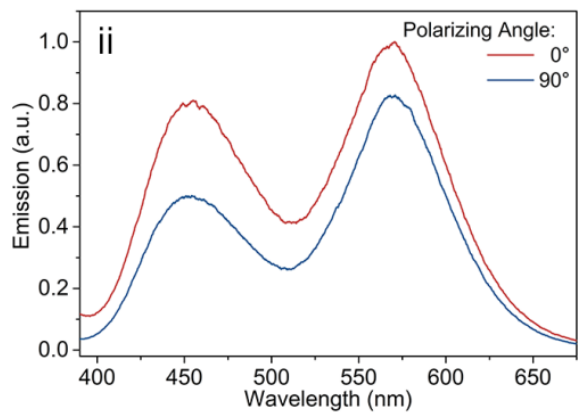

Figure S6. (a) Schematic diagram of the components configuration for polarized emission detection inside a PL spectrometer. The polarizing angle of polarizer 1 was fixed at $0^{\circ}$ and that of polarizer 2 was toggled between $0^{\circ}$ and $90^{\circ}$; (b) PL spectra of (i) neat 1 and (b) the WLE mixture acquired at different polarizing angles. 


\subsection{Thermal Stability}

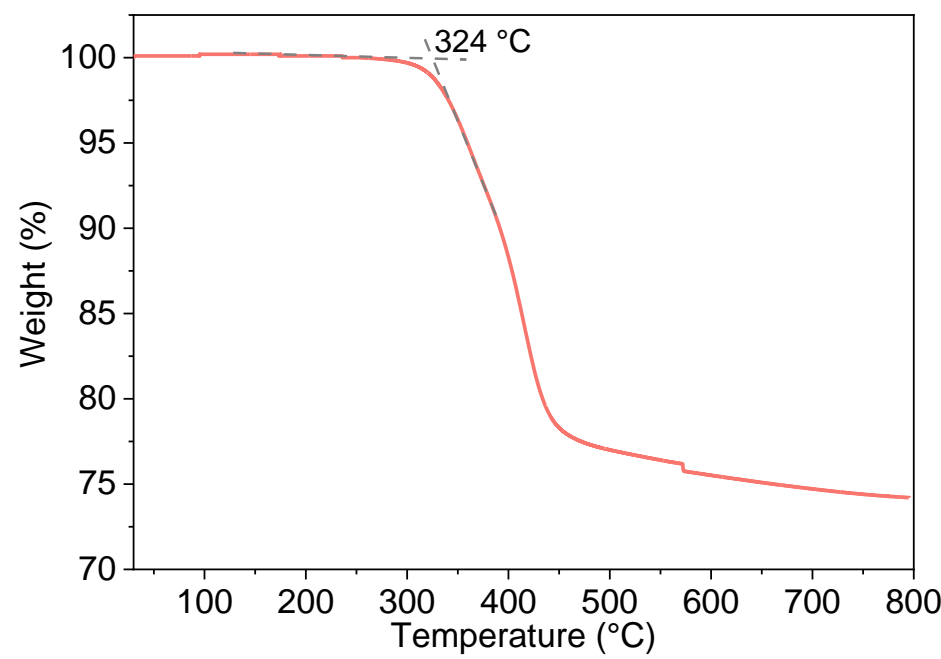

Figure S7. Thermogravimetric diagram of compound 1. 


\subsection{NMR Spectra}

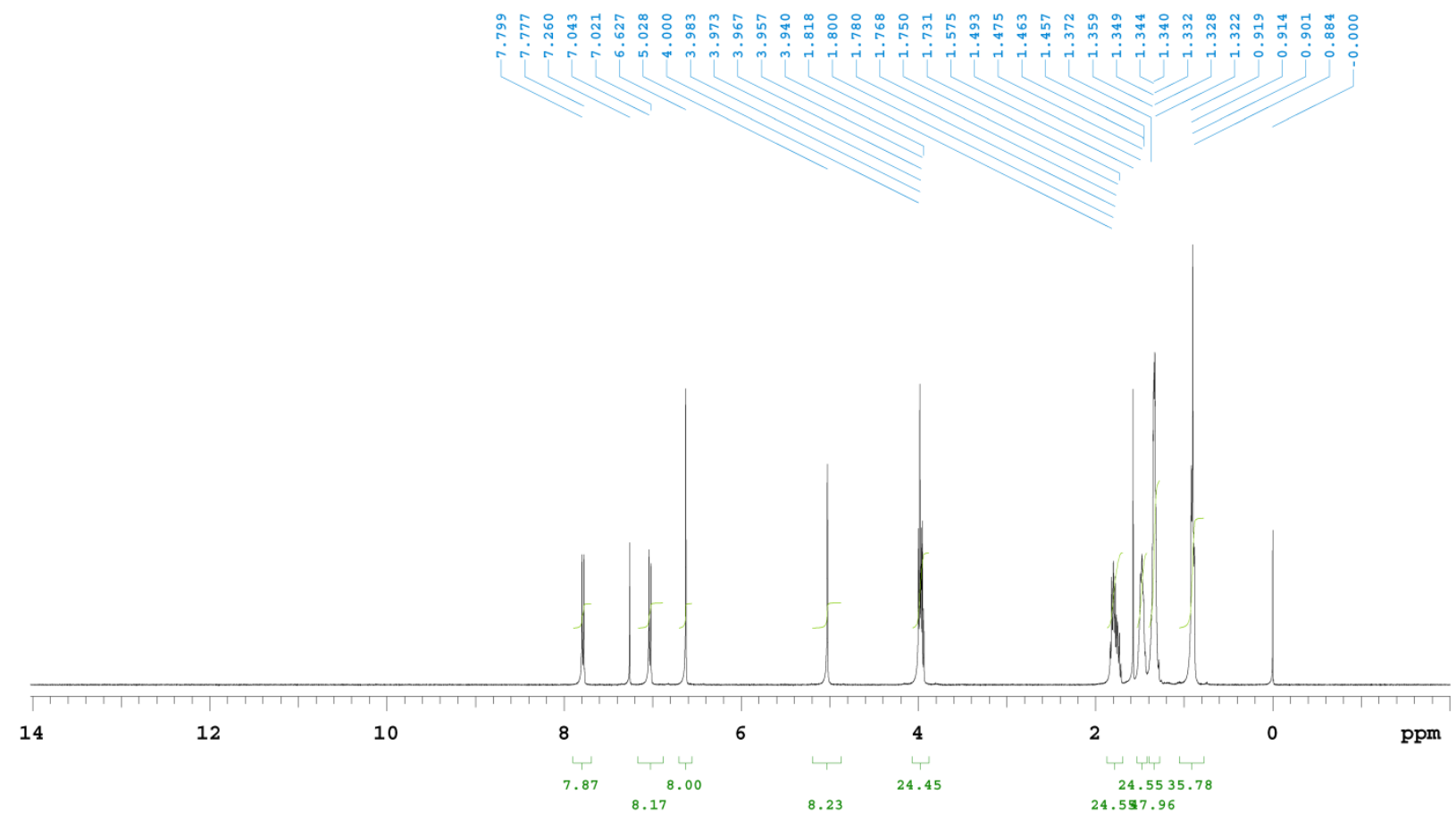

Figure S8. ${ }^{1} \mathrm{H} \mathrm{NMR}(400 \mathrm{MHz})$ spectrum of $\mathbf{K 6}$ in $\mathrm{CDCl}_{3}$.

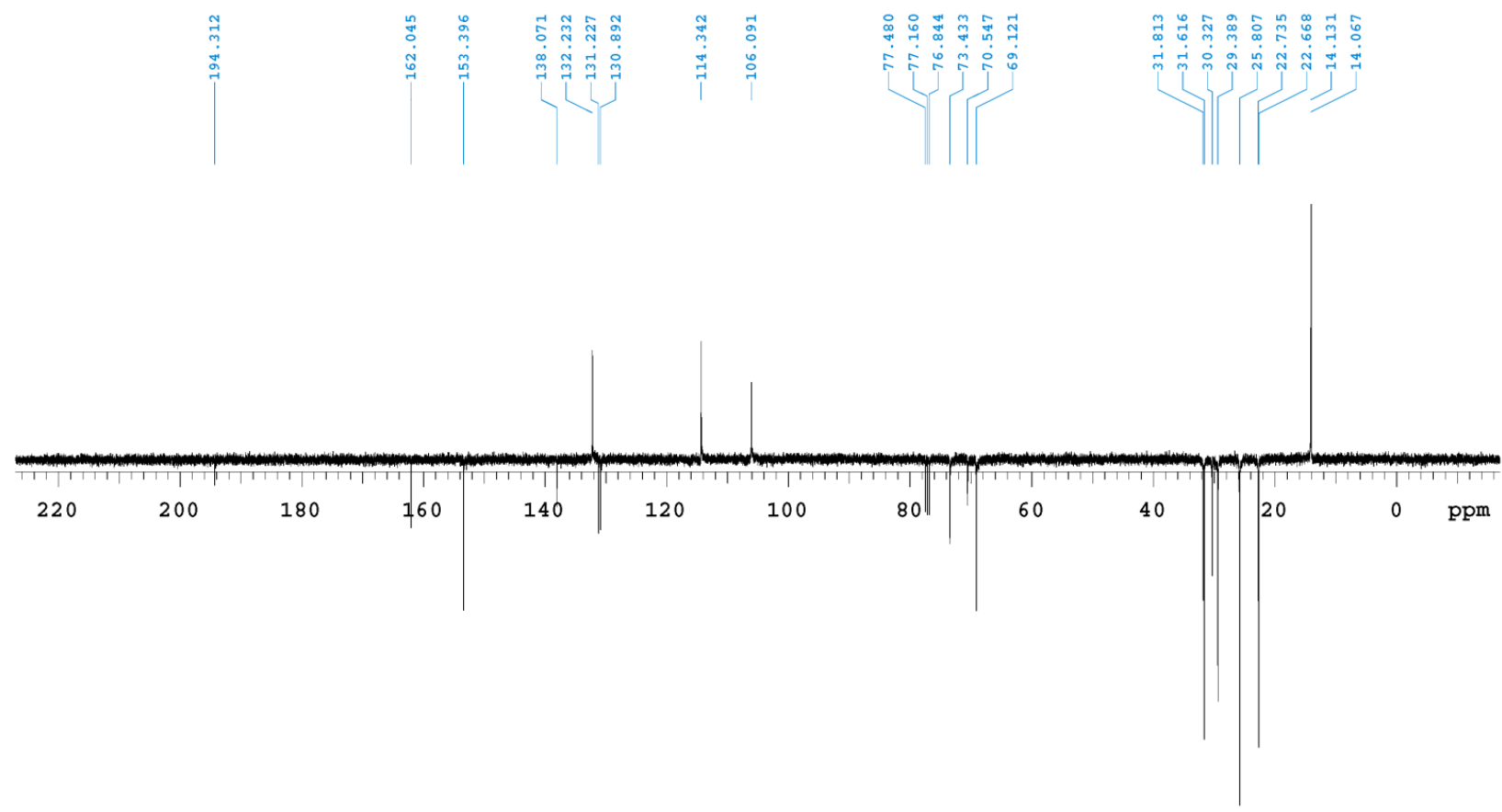

Figure S9. APT (100 MHz) spectrum of $\mathbf{K 6}$ in $\mathrm{CDCl}_{3}$. 


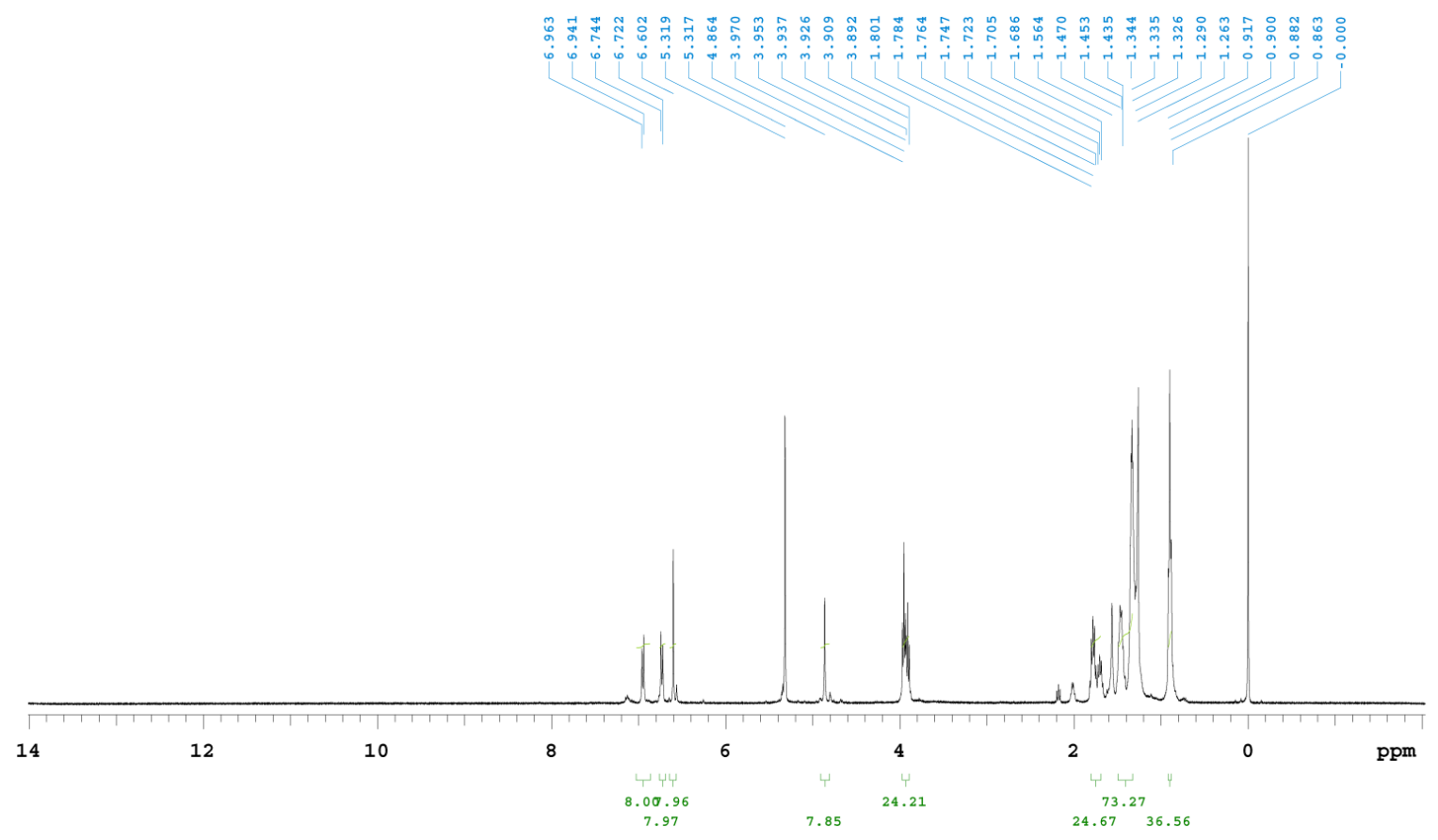

Figure S10. ${ }^{1} \mathrm{H}$ NMR (400 MHz) spectrum of 1 in $\mathrm{CD}_{2} \mathrm{Cl}_{2}$.

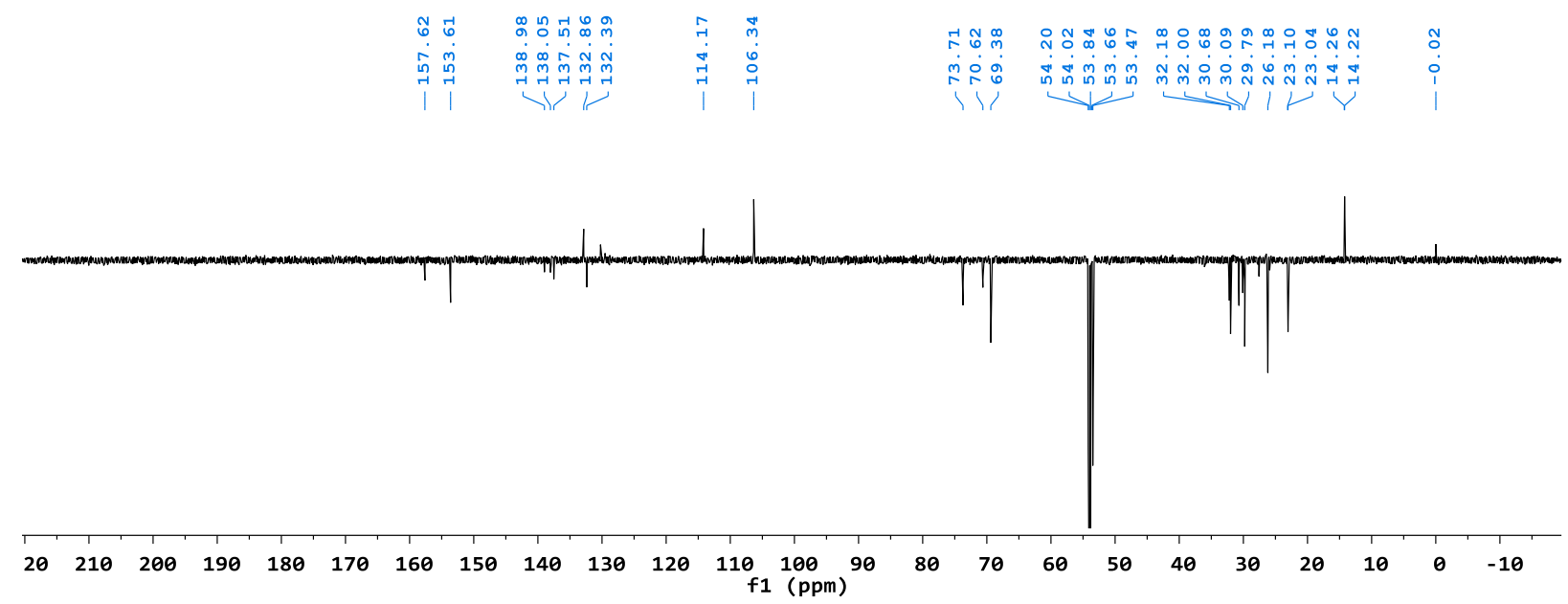

Figure S11. APT (100 MHz) spectrum of 1 in $\mathrm{CD}_{2} \mathrm{Cl}_{2}$. 


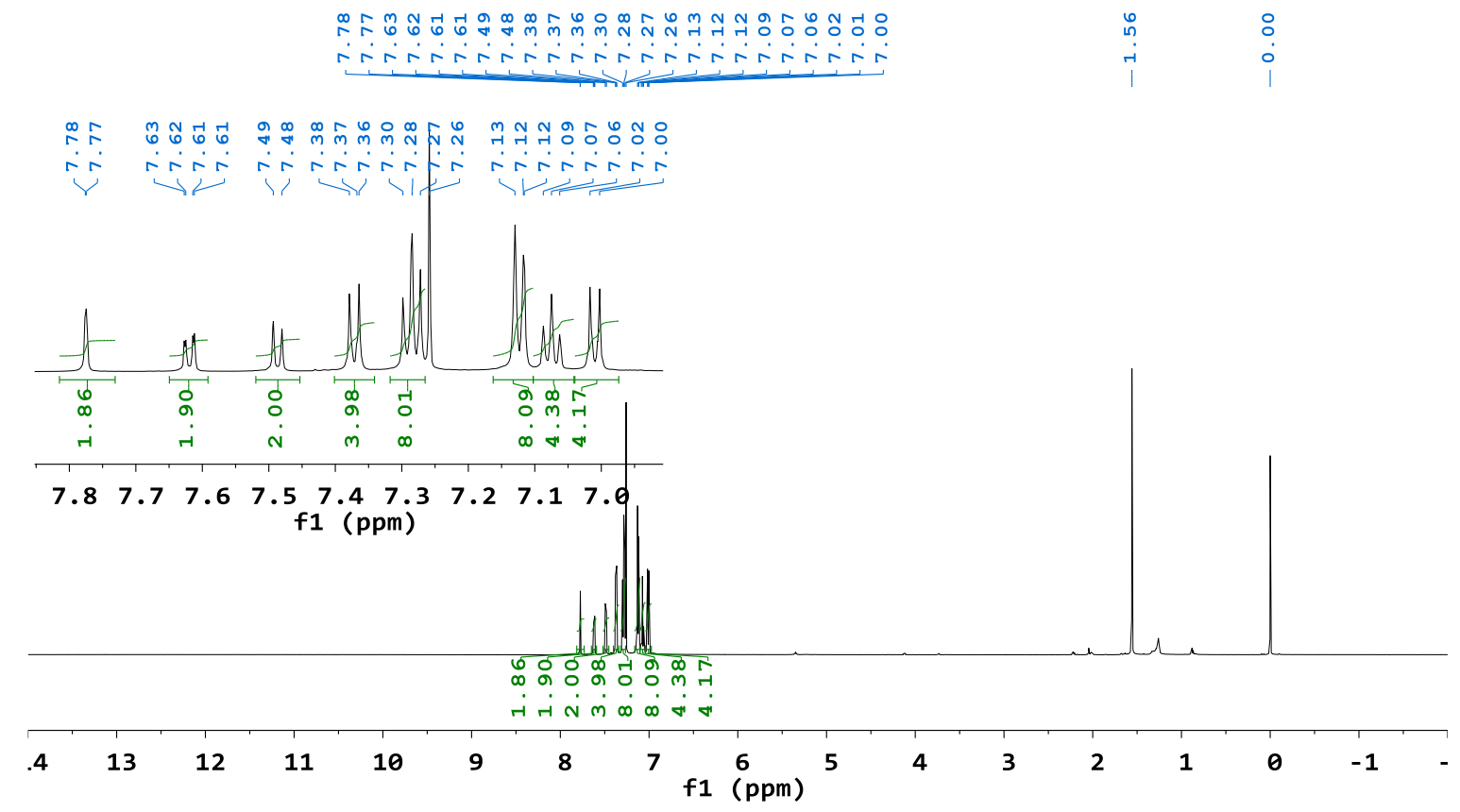

Figure S12. ${ }^{1} \mathrm{H}$ NMR (600 MHz) spectrum of 2 in $\mathrm{CDCl}_{3}$.

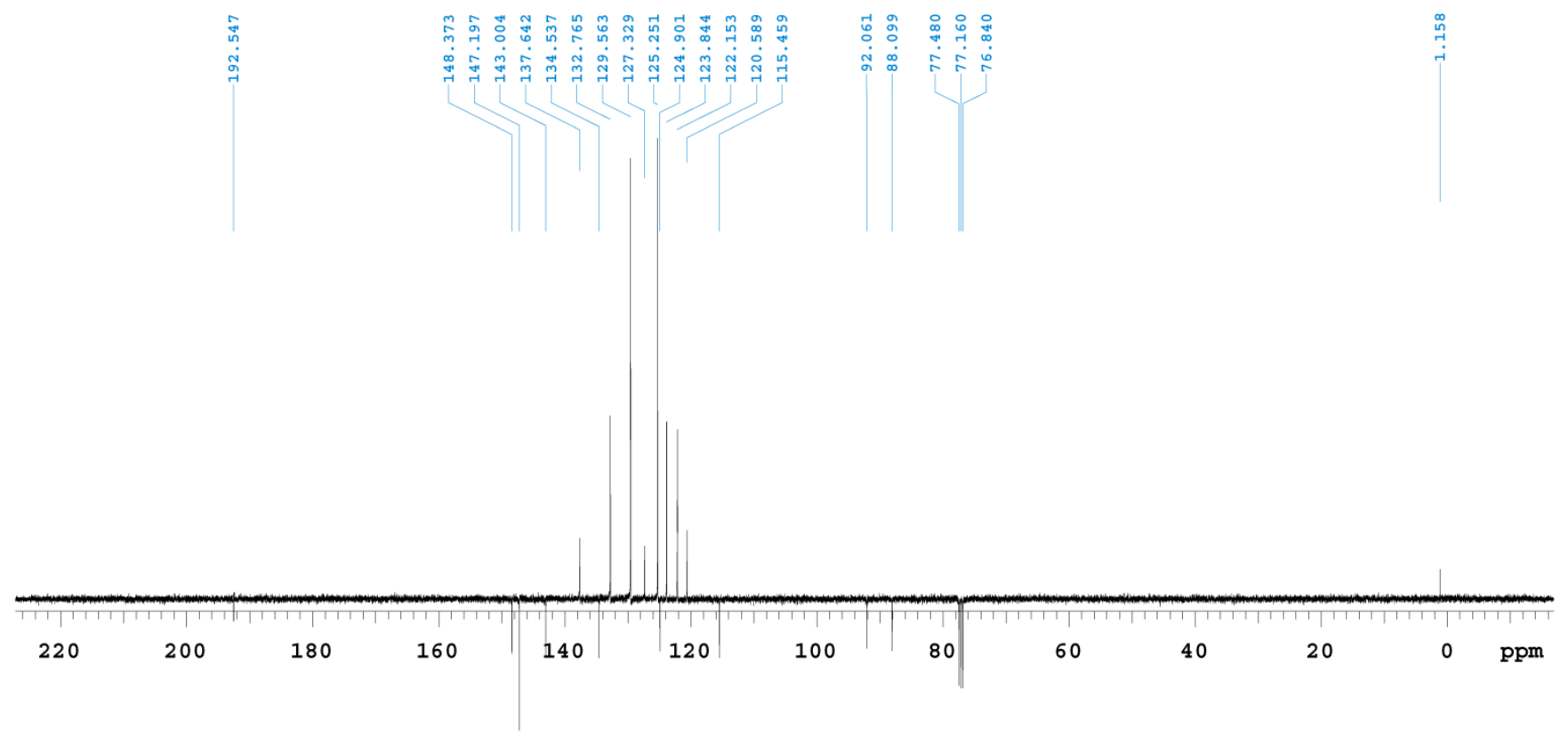

Figure S13. APT $(100 \mathrm{MHz})$ spectrum of 2 in $\mathrm{CDCl}_{3}$. 


\subsection{Mass Spectra}

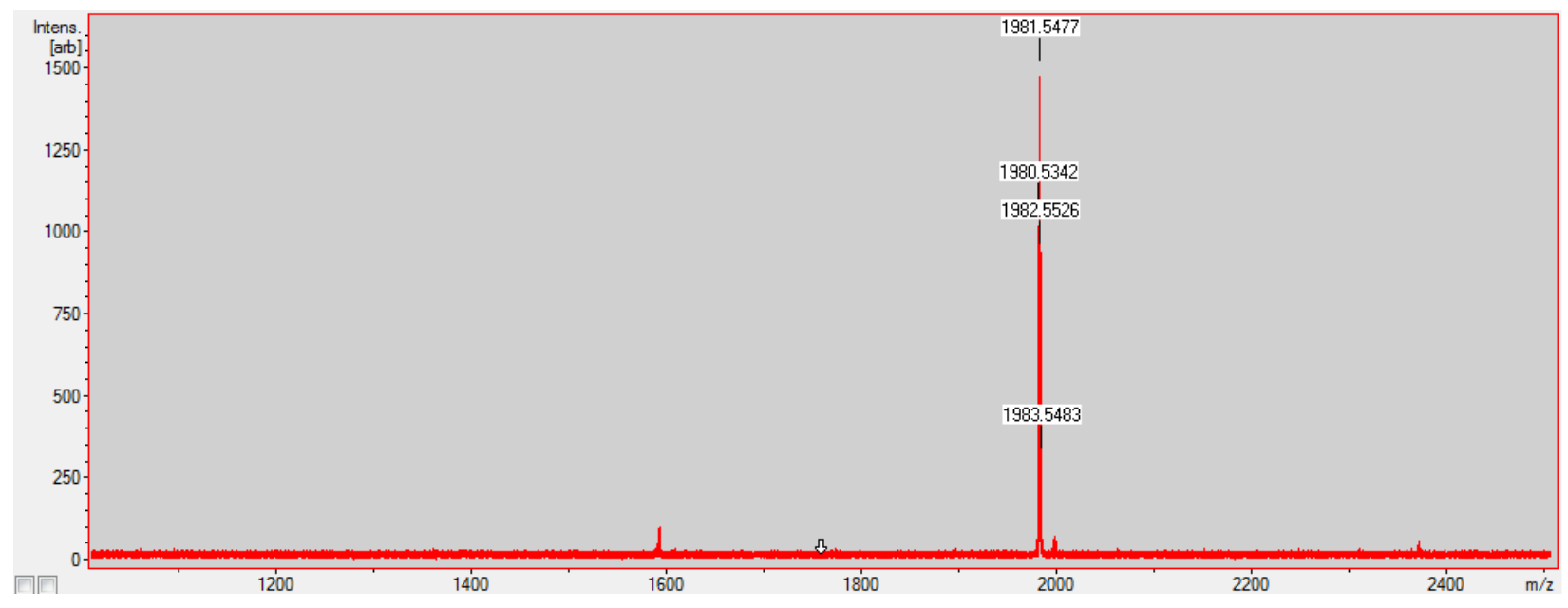

Figure S14. MALDI-TOF mass spectrum of 1.

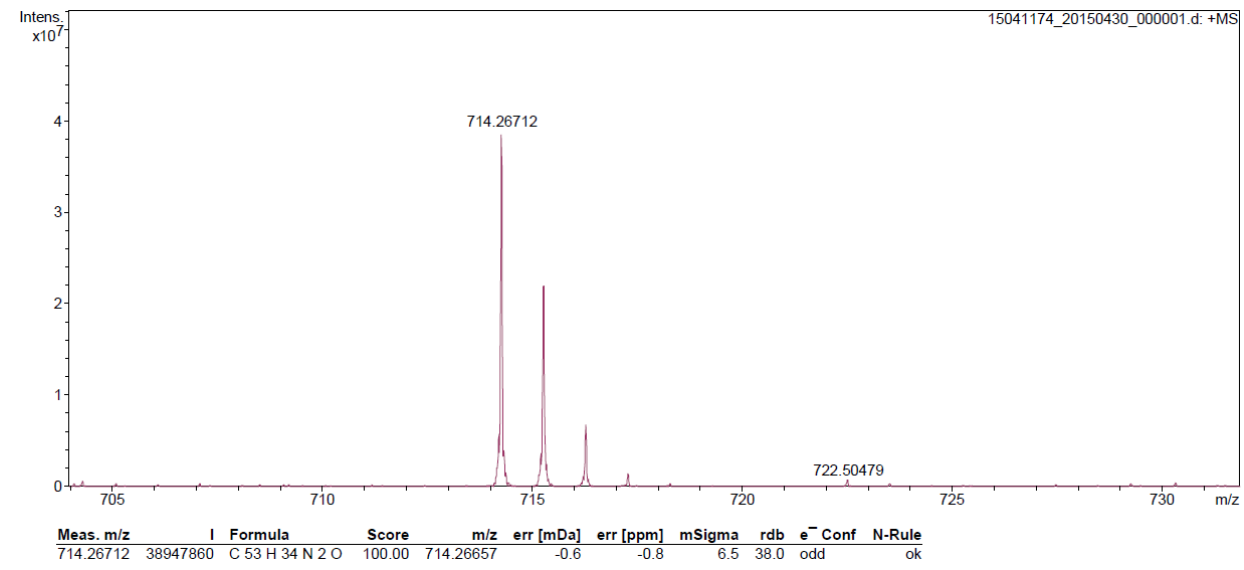

Figure S15. High-resolution ESI mass spectrum of 2. 


\section{References}

1. Fulmer, G. R.; Miller, A. J. M.; Sherden, N. H.; Gottlieb, H. E.; Nudelman, A.; Stoltz, B. M.; Bercaw, J. E.; Goldberg, K. I., NMR Chemical Shifts of Trace Impurities: Common Laboratory Solvents, Organics, and Gases in Deuterated Solvents Relevant to the Organometallic Chemist. Organometallics 2010, 29, 2176.

2. Hundertmark, T.; Littke, A. F.; Buchwald, S. L.; Fu, G. C., $\mathrm{Pd}(\mathrm{PhCN})_{2} \mathrm{Cl}_{2} / \mathrm{P}(t-\mathrm{Bu})_{3}$ : A Versatile Catalyst for Sonogashira Reactions of Aryl Bromides at Room Temperature. Org. Lett. 2000, 2, 1729. 